Joanna Gadacz

Università della Slesia

https://doi.org/10.18778/8220-506-0.16

\title{
UNO SGUARDO FRASEODIDATTICO SULL'ITALIANITÀ, CIOÈ IN QUALE MODO L'APPRENDIMENTO DELLE COLLOCAZIONI ITALIANE DEI COLORI PUÒ AIUTARE A SCOPRIRE LA MENTALITÀ ITALIANA
}

Riassunto: Il lavoro dimostra alcune definizioni e classificazioni delle collocazioni, la loro utilità nel contesto didattico, l'analisi di esercizi lessicali presenti nei manuali di lingua italiana per stranieri ed esamina le collocazioni di colori in base al concetto della mentalità.

Parole chiave: collocazione, manuali d'italiano, mentalità, colori.

\begin{abstract}
A phrase-didactic look at Italianness. How learning the Italian collocations of colors can help to discover the Italian mentality. This paper describes the definitions of collocations and their classification. It presents the utility of collocations in the didactic context, the analysis of the lexical exercises in the Italian textbooks for foreign students and it examines the colour collocations based on the concept of mentalità.
\end{abstract}

Keywords: collocation, Italian textbooks, mindset, colors.

\section{Introduzione}

La fraseodidattica, chiamata anche la didattica della fraseologia, è una disciplina che mira al miglioramento della competenza fraseologica nella lingua materna e il raggiungimento di tale competenza in una lingua straniera (Sułkowska 2013a: 234, 238-239; Sułkowska 2018: 170). In più, si potrebbe dire che questa disciplina pone particolare attenzione alle difficoltà legate all'insegnamento delle espressioni fraseologiche. La fraseologia, costituente un settore della lessicologia, esamina e descrive le combinazioni fisse delle parole 
(chiamate, a seconda della definizione, idiomi o espressioni fraseologiche) i cui significati non derivano né dalla somma dei componenti, né dai loro significati analizzati separatamente. Inoltre, questa analizza anche le combinazioni con il significato globale derivante dalla somma dei significati degli elementi nel caso in cui almeno un componente è stato usato nel modo peculiare o non tipico (Bąba 1986: 8-9). Comunque, non si può sorvolare sul fatto che l'ambito della fraseologia è stato notevolmente esteso e oggi non contiene esclusivamente le espressioni fraseologiche, gli idiomi, i modi di dire, ma anche le diverse combinazioni fisse delle parole che hanno il carattere riproduttivo e non sono create ogni volta durante il discorso, ossia le collocazioni, i termini specialistici, gli indicativi fraseologici ecc. Occorre ribadire che in questa prospettiva della fraseologia essa costituisce un'alta percentuale delle nostre parlate. Secondo le ricerche circa 70\% (Hill 2000: 53) delle produzioni di parlanti nativi comprendono le collocazioni. Perciò diventano un elemento rilevante nello sviluppo della competenza comunicativa. Bisogna prestare l'attenzione al fatto che la formazione delle collocazioni non dipende esclusivamente dal sistema linguistico, bensì è una scelta arbitraria di una comunità parlante che preferisce una certa combinazione di parole più di un'tra che è equivalente e grammaticalmente corretta (in Tornel, Bernal 2017: 204). Dunque, analizzando le tendenze nell'uso delle collocazioni si può avvicinare alla questione della mentalità, cioè, citando il dizionario Treccani al "modo particolare di concepire, intendere, sentire, giudicare le cose, ritenuto proprio di un individuo, di un gruppo sociale o addirittura di un popolo"1.

In questo lavoro si vuole presentare perché l'insegnamento delle collocazioni è fondamentale nell'apprendimento della lingua italiana e, in più, provare a collegarlo con la mentalità italiana. La presente relazione sarà divisa in tre parti: la prima si focalizzerà sulla questione della definizione e classificazione delle collocazioni, nella seconda sarà dimostrata l'analisi di alcuni libri di testo d'italiano per stranieri. Alla fine, la terza parte sarà dedicata all'aspetto della mentalità, ossia in quale modo le collocazioni di colori si riferiscono alla mentalità italiana.

\section{Collocazioni - definizioni, terminologia, classificazioni}

È opportuno sottolineare che le combinazioni fisse di parole suscitano un particolare interesse dei didattici e dei linguisti già da alcuni decenni. Basti pensare, a titolo di esempio, ai lavori di J.R. Firth (1957) che negli anni 50 ha introdotto il termine "collocazione" oppure all'approccio lessicale di M. Lewis

1 www.treccani.it/vocabolario/mentalita/ [01/03/2020]. 
(1993) che negli anni 90 sottolineava l'importanza delle combinazioni di parole nell'apprendimento. Bisogna rilevare che le ricerche sulle collocazioni sono state ultimamente facilitate grazie allo sviluppo dei corpora lessicali ${ }^{2}$. Per quanto riguarda l'interesse verso le collocazioni in Italia, lì sono stati pubblicati tre dizionari cartacei delle collocazioni italiane: Urzì (2009); Tiberii (2012) e Lo Cascio (2013).

Venendo al nocciolo della questione, si passa ora in rassegna delle definizioni più diffuse del concetto fondamentale per il presente lavoro, cioè della collocazione. Quelle sono specificate, secondo le descrizioni più semplici, come "combinazioni fisse, o comunque ristrette di unità lessicali" (Corda 1999: 189), "combinazioni ad alta frequenza" (Balboni 2013:39), oppure "parole che si vanno via via formando all' interno del sistema linguistico, gli accoppiamenti privilegiati di parole che si riscontrano abitualmente nell'uso" (Adamo, Della Valle 2008: 8). Inoltre, si ritiene che esse siano "unità sintagmatiche bimembri, generalmente non idiomatiche; autonome, caratterizzate da una restrizione combinatoria che le presenta come formule fisse" (Partesotti, Tosi 2007: 272) e che

le collocazioni conferiscono un carattere di prevedibilità al significato in base alla compatibilità fra le parole (certe parole si presentano con frequenze verificabili e prevedibili accanto ad altre di un certo tipo) consentendo di individuare anche nel lessico aspetti sistematici, che non rivestono il carattere di vere e proprie "regole" ma almeno quello di "tendenze" (cit. Serra Borneto 1998b: 231-232).

Secondo la denominazione di F.J. Hausmann (2004) la collocazione è una combinazione di parole in cui il primo elemento mantiene il suo senso comune, abituale ed è chiamato base. Un altro elemento, invece, è un collocativo che è scelto con lo scopo di esprimere un dato significato. È importante evidenziare che esso è spesso trasparente, tuttavia è difficilmente prevedibile (p.es. blu profondo - blu in questo caso è la base e conserva il senso primario, profondo è il collocativo).

Un argomento di fondo da tener presente qui si riferisce al modo con cui le collocazioni nascono in una comunità parlante. Non si può sorvolare sul fatto che le collocazioni non sono limitate dalle restrizioni semantiche previste dal sistema linguistico, ma piuttosto dalle "convenzioni e idiosincrasie tipiche dell'uso della singola lingua e del 'costume linguistico' [...] che danno luogo ovviamente ad associazioni preferenziali di lessemi ricorrenti in dipendenza dei diversi scenari rappresentati e degli oggetti e concetti in essi 'attivi'” (cit. Berruto,

2 CoLFIS (1992-1994), CORIS (iniziato nel 1998), L.A.I.C.O. (iniziato nel 2005), LIP (1993), LIT (2006), PEC (2014). 
Cerruti 2011:201-202). Tutto ciò significa che le collocazioni si caratterizzano per un alto grado di arbitrarietà, ciò presuppone le difficoltà durante il loro l'apprendimento in una lingua straniera.

Discutendo la questione delle collocazioni non si può dimenticare i problemi terminologici legati alla distinzione della collocazione e dell'unità polirematica ${ }^{3}$. Per definire la polirematica ci si riferisce ai lavori di De Mauro (LIP; GRADIT) ${ }^{4}$ da cui risulta che queste espressioni si caratterizzano dell'agrammaticalità, della non sostituibilità, non modificabilità e non composizionalità (cfr. Squillante 2016: 4-12).

Si passa ora ad alcune classificazioni delle collocazioni che si potrebbero definire secondo tre tipi: testuale (un'occorrenza di due o più parole che sono vicine una all'altra in un testo), statistico (il rapporto di un'unità lessicale con unaltra unità che appare con una probabilità più che rara in un contesto) e psicologico (le aspettative dei parlanti nativi giocano un ruolo importante nella formazione delle collocazioni) (cfr. Nuccorini, Pinnavia 2008: 75-76).

Inoltre, da unaltra prospettiva si possono distinguere quelle fisse, ossia rigidamente istituzionalizzate (p.es. le espressioni idiomatiche, tipo avere un diavolo per capello) e semifisse, più libere, che consentono le combinazioni a livello sintagmatico, seppure in modo limitato (p.es. il calo / l'aumento / la riduzione dei prezzi) (Menegazzo 2006: 69-70).

Continuando il nostro discorso è importante soffermarsi sulla classificazione delle collocazioni proposta da F. Grossmann e A. Tutin (in Sułkowska 2013: 45-47). Sulla base della fissazione e dell'idiomaticità del collocativo questi studiosi hanno differenziato le collocazioni opache, trasparenti e regolari. Cominciando dalle collocazioni opache occorre sottolineare che il collocativo di queste è imprevedibile e non motivato semanticamente. Dunque, tale collocazione è difficilmente decodificabile e completamente imprevedibile. Le collocazioni trasparenti, invece, hanno il collocativo facilmente comprensibile, ma imprevedibile dal punto di vista lessicale e/o sintattico. Alla fine, le collocazioni regolari sono combinazioni o sequenze i cui significato è generalmente deducibile e sempre prevedibile, benché le regole di associazione siano a volte complesse ed elaborate.

3 Gli altri termini usati alternativamente con la polirematica sono: composto sintagmatico (Scalise 1994), espressione idiomatica (Vietri 1985), lessema complesso (Voghera 1994), espressione multiparola (Masini 2007), parola sintagmatica (Masini 2007).

4 Tullio De Mauro et al. (1993), LIP Lessico di frequenza dell'italiano parlato, Milano, Etaslibri.

Tullio De Mauro (1999-2007), GRADIT - Grande Dizionario Italiano dell'Uso, Torino, UTET. 


\section{Parte didattica}

È opportuno indicare perché l'insegnamento delle collocazioni può essere considerato essenziale nell'apprendimento di una lingua straniera. Prima di tutto, si considera che proprio la conoscenza del lessico possa garantire il successo nella comunicazione (Baldi 2008: 7; Menegazzo 2006: 75, 88; Spreafico 2003: 65). Ovviamente, per comunicare non basta la memorizzazione di un elenco di 3000 parole usate più spesso, bensì bisogna sapere in quale modo è possibile collegare le parole, cioè il comportamento collocazionale. In più, le ricerche indicano che, parlando, usiamo i blocchi di significato costituiti da 4 a 10 parole per rendere il discorso scorrevole e ridurre lo sforzo cognitivo (Menegazzo 2006: 68). Perciò l'insegnamento delle collocazioni può essere un grande d'aiuto per gli apprendenti e diventa chiaro perché le difficoltà nella comunicazione sono dovute in maggior parte alle difficoltà lessicali.

Bisogna porre attenzione al fatto che le collocazioni sono così importanti nell'apprendimento di una lingua straniera che il loro valore è stato segnalato al livello istituzionale (Partesotti, Tosi 2007: 271). Il QCER, anche se non distingue separatamente la competenza collocazionale, adotta la concezione allargata del lessico, cioè non come una categoria comprendente esclusivamente le parole isolate e singole, bensì pure le diverse espressioni fisse o cristallizzate, cioè tutte quelle forme che si apprendono come un insieme unico (Diadori 2009: 150). Per quanto concerne a quale livello si dovrebbe conoscere quelle espressioni fisse, il QCER indica che certe strutture principali dovrebbero essere presenti già al livello $\mathrm{A}$, specialmente $\mathrm{A} 2$. Ai livelli più avanzati: $\mathrm{B} 2, \mathrm{C} 1$ e C2 è auspicabile non solo la conoscenza di tali unità lessicali, ma anche la capacità di riflettere sui loro usi, sulle modificazioni che subiscono frequentemente e sul loro comportamento collocazionale (Corda 1999: 45; Sułkowska 2018: 172; Partesotti, Tosi 2007: 273).

Ora ci si concentrerà sull'analisi di alcuni manuali di lingua italiana per stranieri dedicati ai livelli B2-C2 poiché la conoscenza delle collocazioni è richiesta a tali livelli (come è già stato menzionato prima). Lo scopo dell'analisi è prestare l'attenzione al fatto se esse siano veramente presenti, in quale tipo di esercizio e a quali altre attività si riferiscono tali esercizi, cioè se le collocazioni sono presentate con o senza contesto. In più, si deve evidenziare che saranno esaminati gli esercizi in cui le collocazioni sono sottolineate e ben visibili. Si pone una particolare attenzione alle collocazioni costruite in base allo schema verbo + nome / aggettivo / avverbio.

Dopo aver svolto l'analisi di dieci manuali di lingua italiana agli stranieri (i titoli si trovano in sezione Bibliografia) ci si focalizza su due manuali: Contatto e Magari. 
Nel Contatto gli esercizi si concentrano prima di tutto sulla comprensione scritta e orale, ma sono presenti anche tanti esercizi lessicali, p.es. su sinonimi, contrari, parafrasi, famiglie di parole, derivazione, verbi pronominali idiomatici, espressioni idiomatiche, nomi composti e metafore. Il particolare interesse è stato suscitato dagli esercizi in cui è analizzato il lessico e la grammatica simultaneamente: in cinque su sei unità sono presenti gli esercizi sulle collocazioni che richiedono l'uso delle preposizioni giuste. Bisogna mettere in risalto il fatto che gli esercizi lessicali si riferiscono quasi sempre alle altre attività che prevedono lo sviluppo delle competenze diverse, p.es ascolto, lettura, produzione orale.

Magari è un corso di lingua italiana per stranieri rivolto a studenti di livello intermedio e avanzato (dal B1 al C1). Nell'introduzione si sottolinea che "un importante rilievo è $[\ldots]$ dato allo studio del lessico $[\ldots]$ accogliendo alcune suggestioni dell'approccio lessicale". Il volume comprende ventuno unità didattiche, che non si dividono in sezioni, bensì sono costituite dagli esercizi diversificati dedicati allo sviluppo di varie competenze. Bisogna evidenziare la presenza di molte attività ludiche (giochi a squadre, cruciverba), ma soprattutto il modo di presentazione degli esercizi lessicali: tutti gli esercizi lessicali sono collegati con la lettura del testo, cioè tutte le espressioni evidenziate sono contestualizzate. Inoltre, essi riguardano una vasta gamma di diversi aspetti lessicali, p.es. sinonimi, contrari, derivazione, genere, parole composte, verbi pronominali, ma soprattutto le collocazioni che sono state nominate nell'indice. Inoltre, le collocazioni sottolineate non basano soltanto sullo schema verbo + nome / aggettivo / avverbio, ma anche nome + aggettivo.

Dopo aver presentato questa breve panoramica si è inclini ad appoggiare la tesi sostenuta da M. Partesotti e R. Tosi (2007: 270) che evidenziano che, sebbene le molte indicazioni e raccomandazioni, le unità fraseologiche non sono spesso considerate oggetto di studio e di programmazione didattica poiché non tutti i libri prevedono il lavoro su di esse. All'insegnamento del lessico si dedicano spazi nella manualistica, anche se non sempre organizzati, tuttavia in pochi manuali troviamo riferimenti organici a collocazioni: soltanto due su dieci libri le nominano. Inoltre, agli esercizi lessicali spesso manca il contesto il che rende lo studio della fraseologia più difficile. Per quanto attiene agli esercizi dedicati allo sviluppo della competenza collocazionale, questi sono pochi, consistono, soprattutto, nel collegamento dell'espressione e del suo sinonimo oppure nella scelta multipla e mancano gli esercizi focalizzati sullo sviluppo delle competenze attive. Si può presumere anche che i manuali con degli esercizi sulle collocazioni saranno sempre più richiesti sul mercato, considerando il crescente interesse nel campo di fraseodidattica. 


\section{Analisi di collocazioni di colori in lingua italiana}

Nella parte analitica del presente articolo saranno dimostrate le collocazioni di colori individuate in base al dizionario delle collocazioni a cura di Paola Tiberii: Dizionario delle collocazioni. Le combinazioni delle parole in italiano dal 2012. Il corpus è costituito da 192 collocazioni di colori come azzurro, bianco, blu, giallo, grigio, rosa, rosso, marrone, nero. Bisogna sottolineare che nel dizionario non si trovano le collocazioni di colori arancione e viola. Dopo aver estratto le locuzioni, queste sono state divise in cinque categorie descriventi attraverso quali riferimenti con il mondo gli italiani concepiscono i colori. Nell'elenco ci sono le collocazioni formate combinando NOME (colore) + AGGETTIVO e NOME (colore) + NOME e le locuzioni o modi di dire chiamati nel dizionario costruzioni.

La prima categoria contiene le qualità dei colori, cioè gli aggettivi che si riferiscono all'intensità, alla luminosità, tonalità, purezza e trasparenza. Per quanto riguarda l'intensità quasi tutti i colori possono essere descritti con l'aggettivo intenso (azzurro / blu / giallo / marrone / nero / rosa / rosso / verde intenso). Ci sono anche molti esempi di collocazioni con l'aggettivo pallido (azzurro / giallo / rosa / verde pallido). I colori giallo, rosso e verde appaiono anche con carico (giallo / rosso / verde carico). Invece l'aggettivo sbiadito si unisce con il nero e il verde (nero / verde sbiadito) e solo l'azzurro forma una collocazione con l'aggettivo slavato (azzurro slavato). Un'altra caratteristica, cioè luminosità è presente nelle collocazioni seguenti: verde / nero lucente, azzurro / giallo / marrone luminoso, bianco / giallo lucido, azzurro / giallo / nero / rosa / rosso / verde / brillante. Per quanto riguarda la tonalità dei colori, la chiarezza è definita con l'unico aggettivo chiaro (azzurro / giallo / grigio / marrone / rosa / rosso / verde chiaro) mentre il contrario è espresso con due aggettivi: scuro (blu / giallo / grigio / marrone / verde scuro) e cupo (rosso / verde cupo). L'aspetto della purezza si riferisce esclusivamente al bianco (bianco sporco / candido) e per indicare la trasparenza si usa un aggettivo opaco (bianco / giallo / nero opaco). Va menzionato che ci sono molte collocazioni che si riferiscono a un altro colore, p.es. azzurro glauco / verdastro / violaceo, grigio / verde azzurro, nero bluastro, verde giallo, grigio verde.

Il secondo gruppo di aggettivi e nomi descriventi i colori in lingua italiana presenta le associazioni con gli elementi, cioè con il fuoco, l'acqua, e la terra. I riferimenti al fuoco si osserva nei casi seguenti: rosso fuoco, rosso fiammante, marrone bruciato, giallo / rosso acceso (che brucia, riscalda come il fuoco) e al contrario azzurro / rosso / verde spento. Due elementi inseparabili dal fuoco sono il fumo è il cenere. Il fumo forma una collocazione grigio fumo e una locuzione grigio fumo di Londra. Il cenere si vede invece in: azzurro / grigio polvere, grigio cenere. Le collocazioni con l'acqua si riferiscono a due stati di 
acqua, cioè allo stato liquido e solido; azzurro / blu oltremare, verde acqua, blu mare / marino ovviamente indicano lo stato liquido e lo stato solido è usato in collocazioni come azzurro / bianco ghiaccio, bianco niveo (si riferisce alla neve). L'elemento di terra è presente in solo una collocazione giallo ocra (l'ocra è una varietà di terra argillosa).

La terza categoria basa sulla materia non vivente. Le collocazioni come azzurro / blu cielo indicano una parte dell'atmosfera. Comunque, il gruppo più frequente di questa classe è formato attraverso l'utilizzo di un elemento chimico (che è simultaneamente anche il metallo): azzurro / blu cobalto, bianco argenteo, giallo / marrone dorato, giallo oro, grigio argenteo / argento / ferro, grigio piombo. Si distinguono pure le espressioni con le rocce: azzurro / grigio ardesia, bianco gesso, grigio / nero antracite (l'antracite è un carbone fossile).

Ora si passa all'ambiente vivente utilizzato nella descrizione dei colori. $\mathrm{Ci}$ sono molte collocazioni che si riferiscono alle piante: azzurro glicine / pervinca, rosa fucsia, rosso geranio, verde erba, verde malva, verde muschio, verde oliva. Inoltre, si trovano gli esempi di collocazioni con la frutta: giallo limone, rosso ciliegia, verde mela / pistacchio (il pistacchio compreso come la frutta dell'albero di pistacchio). Tranne le collocazioni con l'aggettivo nel dizionario si trova anche una locuzione marrone testa di moro. Fra gli altri esempi, si osserva un riferimento alla verdura verde pisello. Invece la collocazione verde marcio indica una qualità della frutta e verdura. In più, bisogna porre attenzione alle collocazioni animalesche. Nel dizionario si trovano casi con degli uccelli: blu / verde pavone, giallo canarino, nero corvino, con un pesce rosa salmone, con un corallo rosa / rosso corallo, con un verme rosso vermiglio e con un mammifero grigio topo. In più ci sono le collocazioni con gli aggettivi riguardanti la vita e la vivacità: azzurro / verde / rosso / giallo vivo, giallo / rosso vivace. C’è solo un esempio di un fluido presente nell'essere umano e negli animali, cioè rosso sangue.

L'ultima categoria si riferisce all'attività dell'uomo in ampio contesto. In molti casi si osserva l'uso dei sensi come tocco, vista e udito. Il tocco si vede nelle collocazioni azzurro / verde / rosa / giallo tenue, marrone caldo, rosa delicato perché per dire che qualcosa è delicato, bisogna, senza dubbi, toccarlo prima. $\mathrm{Si}$ ha a che fare, quindi, con il fenomeno di sinestesia. Le collocazioni come bianco / giallo abbacinante, bianco abbagliante, bianco / giallo accecante, rosso / verde sgargiante (vistoso, appariscente) presentano l'uso della vista. Queste espressioni dimostrano le associazioni negative, quando un colore è così visibile che fa male. Per quanto riguarda l'udito, nel dizionario si trova un esempio della collocazione rosso squillante.

Continuando il discorso si vuole segnalare i casi di collocazioni con i materiali usati dall'uomo, per esempio: bianco avorio / eburneo, bianco porcellanato, grigio acciaio / metallizzato. Poi, è tipico per l'uomo indossare 
gioielli i quali hanno molte collocazioni: grigio madreperlato / perla, giallo ambra / ambrato, rosso rubino, verde smeraldo. Gli altri casi particolari che suscitano l'interesse sono le espressioni con i riferimenti militari come blu avio (di tonalità di blu usata per le divise militari dell'aeronautica), verde militare o una locuzione con un'arma: grigio canna da fucile. Un'altra attività dell'uomo, cioè la pittura fornisce le collocazioni con i riferimenti a delle forniture per disegno: azzurro / giallo / verde pastello e i coloranti/pigmenti come azzurro ceruleo, rosso carminio / porpora. Anche le spezie formano le collocazioni: giallo zafferano, verde salvia. Inoltre, attirano l'attenzione le collocazioni che sono difficili da classificare in una categoria che si riferiscono ai diversi aspetti dell'attività dell'uomo che sono elencati sotto:

- rosso cardinale (posizione, carica),

- giallo senape, bianco latte (prodotto alimentare),

- rosso lacca (sostanza non alimentare),

- giallo paglierino (prodotto agricolo),

- rosso mattone (materiale utilizzato nell'edilizia),

- rosso pompeiano (città, luogo),

- blu petrolio (sostanza),

- blu notte (parte del giorno),

- verde bottiglia (contenitore),

- rosa antico (epoca),

- rosa cipria (cosmetico),

- azzurro / rosa confetto (dolce),

- bandiera verde (riconoscimento di una località marina),

- locuzione bianco e nero,

- locuzione azzurro carta da zucchero (aspetto culturale - identificata con la nascita di un bambino maschio).

Alla fine, sarebbe opportuno indicare anche le collocazioni con la comprensione metaforica: azzurro / nero profondo, bianco / nero assoluto, bianco immacolato, blu elettrico nero impenetrabile / totale.

\section{Conclusioni}

Dal breve quadro teorico riguardante le posizioni si può concludere che il termine collocazione, anche se conosciuto da circa 70 anni, non possiede una definizione chiara e ben stabilita. Comunque, bisogna evidenziare che le combinazioni fisse di parole sono usate molto spesso nelle conversazioni e in questo modo diventano un elemento necessario nell'apprendimento di una lingua straniera. 
Poichéle collocazioni sono espressioni che si caratterizzano dalla frequenza d'uso e costituiscono una scelta arbitraria di una comunità parlante, queste possono fornire un'indicazione nella ricerca della mentalità di una nazione.

Dall'analisi delle collocazioni dei colori presenti in lingua italiana risulta che gli italiani concepiscono i colori tramite cinque categorie principali, ossia: 1) qualità dei colori come intensità, luminosità, tonalità, purezza e trasparenza; 2) associazioni con gli elementi come fuoco, acqua, terra; 3 ) materia non vivente come atmosfera, metallo, roccia; 4) ambiente vivente, come piante, frutta, animali; 5) attività dell'uomo in ampio contesto, per esempio il materiale utilizzato o i gioielli.

Alla fine, bisogna enfatizzare che sarebbe conveniente svolgere una ricerca simile concernente la lingua polacca e in seguito unanalisi contrastiva per trovare le differenze e le similitudini con la mentalità polacca.

\section{Bibliografia}

Adamo, Giovanni e Della Valle, Valeria (2008). Le parole del lessico italiano, Roma, Carocci.

Balboni, Paolo Ernesto (2013). Fare educazione linguistica. Insegnare italiano, lingue straniere e lingue classiche, Torino, UTET.

Baldi, Pier Luigi (2008). Le parole della mente: lessico mentale e processi linguistici, Milano, FrancoAngeli.

Corda, Alessandra e Marello, Carla (1999). Insegnare e imparare il lessico, Torino, Paravia.

Diadori, Pierangela e Palermo, Massimo e Troncarelli, Donatella (2009). Manuale di didattica dell' italiano L2, Perugia, Guerra Edizioni.

Firth, John Rupert (1957). Papers in linguistics 1934-1951, Oxford, OUP.

Hausmann, Franz Josef (2004). "Was sind eigentlich Kollokationen?", [in] Kathrin Steyer (a c. di), Wortverbindungen - mehr oder weniger fest, Institut für Deutsche Sprache Jahrbuch 2003, 2004, pp. 309-334.

Hill, Jimmie (2000). "Revising priorities: From grammatical failure to collocational success", [in] Michael Lewis (a c. di), Teaching collocation: Further developments in the lexical approach, Hove, LTP, pp. 47-67.

Lewis, Michael (1993). The Lexical Approach, Hove, LTP.

Lo Cascio, Vincenzo (2013). Dizionario Combinatorio Italiano, Amsterdam, Philadelphia, John Benjamins Publishing Company.

Masini, Francesca (2007). Parole sintagmatiche in italiano. Tesi di dottorato di ricerca, Università degli Studi di Roma Tre.

Menegazzo, Edoardo (2006). "Lapproccio lessicale di Lewis e la lingua dello studio", [in] Paolo Ernesto Balboni (a c. di), Didattica e linguistica dell' italiano come lingua straniera. Anno IV, numero 12/2006, Perugia, Guerra Edizioni, pp. 67-89.

Nuccorini, Stefania e Pinnavia, Laura (2008). "Lessico: il ruolo della fraseologia", Studi Italiani di Linguistica Teorica e Applicata, 37 (1), pp. 71-89.

Partesotti, Marina e Tosi, Roberta (2007). "Si fa per dire... «La morte» nella fraseologia", Annali dell'Istituto Universitario Orientale. Sezione Romanza, XLIX (1), pp. 269-297.

Scalise, Sergio (1994). Le strutture del linguaggio, Bologna, Il Mulino. 
Serra Borneto, Carlo (1998). "L'approccio lessicale”, [in] Carlo Serra Borneto (a c. di), C'era una volta il metodo: tendenze attuali nella didattica delle lingue straniere, Roma, Carocci, pp. 227-247.

Spreafico, Lorenzo (2003). "Cercando le parole. Strategie di espressione lessicale nell'apprendimento linguistico: il caso dell'italiano L2”, [in] Paolo Ernesto Balboni (a c. di), Didattica e linguistica dell' italiano come lingua straniera. Anno I, numero 3/2003, Perugia, Guerra Edizioni, pp. 65-84.

Squillante, Luigi (2016). Polirematiche e collocazioni dell'italiano. Uno studio linguistico e computazionale. Tesi di dottorato di ricerca, Sapienza Università di Roma e Universitat Hildesheim. hildok.bsz-bw.de/frontdoor/index/index/docId/535 [01/03/2020].

Sułkowska, Monika (2013). De la phraseologiè à la phraséodidactique. Ètudes thèoriques et pratiques, Katowice, Wydawnictwo Uniwersytetu Śląskiego.

Sułkowska, Monika (2018). „Frazeodydaktyka i frazeotranslacja jako nowe dyscypliny frazeologii stosowanej", Applied Linguistics Papers, 25 (2), pp. 169-181.

Tiberii, Paola (2012). Dizionario delle collocazioni. Le combinazioni delle parole in italiano, Bologna, Zanichelli.

Torner, Sergi, Bernal, Elisenda (2017). Collocations and other lexical combinations in Spanish. Theoretical, lexicographical and applied perspectives, London-New York, Routledge.

Urzi, Francesco (2009). Dizionario delle combinazioni lessicali, Lussemburgo, Edizioni Convivium.

Vietri, Simonetta (1985). Lessico e sintassi delle espressioni idiomatiche. Una tipologia tassonomica dell'italiano, Napoli, Liguori Editore.

Voghera, Miriam (1994). “Lessemi complessi: percorsi di lessicalizzazione a confronto”, Lingua e stile, XXIX (2), pp. 185-214.

\section{Sitografia}

www.treccani.it/vocabolario/mentalita/ [01/03/2020].

\section{Corpus}

Tiberii, Paola (2012). Dizionario delle Collocazioni. Le combinazioni delle parole in italiano, Bologna, Zanichelli.

\section{Manuali di lingua italiana per stranieri}

AFFRESCO ITALIANO B2. Corso di lingua italiana per stranieri. Le Monnier. AFFRESCO ITALIANO C2. Corso di lingua italiana per stranieri. Le Monnier. COLORI D'ITALIA. Testi e attività per corsi avanzati. C1-C2. Edilingua.

CONTATTO. Corso di italiano per stranieri. Livello post-intermedio B2. Edizioni Guerra.

MAGARI. Corso di lingua e cultura italiana di livello intermedio e avanzato. B1-C1. Alma Edizioni. MOSAICO ITALIA. Percorsi nella cultura e nella civiltà italiana. B2-C2. Edilingua.

NUOVO PROGETTO ITALIANO 3. Corso multimediale di lingua e civiltà italiana. Livello intermedio - avanzato $(B 2-C 1)$. Edilingua.

NUOVO RETE! B2 Corso multimediale italiano per stranieri. Edizioni Guerra RETE! 3 Corso multimediale d'italiano per stranieri - terzo livello. Libro di classe. Edizioni Guerra. 